\title{
Clinical Manifestations of Myocardial Injury Associated with the 2019 Novel Coronavirus Disease in Chinese Population: A Meta-analysis
}

\author{
Mingyan Gong, Jun Guo* \\ Department of Cardiology, The First Affiliated Hospital of Jinan University, Guangzhou, China \\ Email address: \\ dr.guojun@163.com (Jun Guo) \\ ${ }^{*}$ Corresponding author

\section{To cite this article:} \\ Mingyan Gong, Jun Guo. Clinical Manifestations of Myocardial Injury Associated with the 2019 Novel Coronavirus Disease in Chinese \\ Population: A Meta-analysis. International Journal of Infectious Diseases and Therapy. Vol. 5, No. 4, 2020, pp. 136-144. \\ doi: $10.11648 /$ j.ijidt.20200504.16
}

Received: November 21, 2020; Accepted: December 4, 2020; Published: December 11, 2020

\begin{abstract}
Background: Evidence about COVID-19 on cardiac injury was insufficient. Objectives: To summarize available data on clinical manifestations of acute cardiac injury during the COVID-19 outbreak. Methods: A systematic literature search from December 1, 2019 to April, 12, 2020 was made of those databases: Pubmed, Embase, wanfang, CKNI, to identify all observational studies that reported presentation of cardiac injury, including acute cardiac injury, cardiac symptoms, cardiac failure, shock, arrhythmia. A random-effects model was used to pool results. Heterogeneity was assessed using $\mathrm{I}^{2}$. Publication bias was assessed using Egger's test. Results: 3863 confirmed COVID-19 infected patients from 22 studies were included in the meta-analysis. Among the patients, the incidence rate of acute cardiac injury was $18 \%$ [95\%CI $12-23]$, chest pain (4\% [95\%CI $2-5]$ ), chest distress $(31 \%$ [95\%CI 16-47]), and palpitations (4\% [95\%CI 0-7]) were the most common clinical symptoms related to acute cardiac injury. The incidence with heart failure was $22 \%$ [95\%CI 17-28], the incidence with shock was $9 \%$ [95\%CI 3-14], and the incidence with arrhythmia was 13\% [95\%CI 8-19]. Conclusions: Acute cardiac injury without clinical symptoms should be paid more attention among the confirmed COVID-19 infected patients. In addition, this highlights the need to effectively monitor heart health to prevent the cardiac complications (cardiac failure, shock, arrhythmia) in patients infected with COVID-19.
\end{abstract}

Keywords: COVID-19, Cardiac Injury, Coronavirus, Chest Pain, Acute Cardiac Injury, Cardiac Failure, Shock, Arrhythmia

\section{Introduction}

In December 2019, Wuhan, Hubei first discovered multiple cases of unexplained pneumonia patients. The World Health Organization named the virus as the 2019 new coronavirus pneumonia (COVID-19). Later, the epidemic quickly spread in China and abroad. Researchers have found that 2019-nCoV belongs to the genus $\beta$-coronavirus and was highly contagious. Its mode of spreading was mainly through respiratory tract droplets and close contact with people. In addition to the typical respiratory manifestations, some patients with new coronavirus pneumonia also show clinical manifestations of cardiac involvement. In this study, a meta-analysis of myocardial injury symptoms and comorbidities associated with new coronavirus infection was conducted to further analyze the impact of new coronavirus infection on heart damage.

\section{Material and Methods}

\subsection{Search Strategy}

A systematic literature search from December 1, 2019 to April, 12, 2020 was made of those databases: Pubmed, Embase, wanfang, CKNI. Using the following keywords: Wuhan coronavirus, Wuhan seafood market pneumonia virus, COVID19 virus, COVID-19 virus, coronavirus disease 2019 virus, SARS-CoV-2, SARS2, 2019-nCoV, 2019 novel coronavirus, clinical characteristic. Only available data from published articles were collected. 


\subsection{Inclusion and Exclusion Criteria}

Inclusion criteria: (i). Research type: Include the original documents being analyzed for case-control studies and cohort studies; (ii). Research target: The total number of cases was greater than 20 , and all cased are clearly verified as new coronavirus pneumonia. Exclusion criteria: (i). The study population cannot represent a special sample of the general population; (ii). Repeated report, incomplete information or logical mistakes;(iii). Types of literature such as summary, case report, meeting abstract and etc.

\subsection{Literature Screening and Quality Evaluation}

Read through the title and abstract according to the established inclusion and exclusion criteria, remove relevant documents that do not reach the standsards, and then read through the full text to determine the literature to be included in the study and place it on the data extraction table prepared in advance. Cochrane bias risk assessment tool was used to evaluate the quality of literature. According to the quality evaluation items of the case series of the National Institute of Clinical Optimization in the UK: (i). It was the best for the cases in the case series to be from medical institutions at different levels, which was conducive to improving the representativeness of the research results and conducting multi-center research; (ii). Clearly and accurately describe the purpose and objectives of the study; (iii). Clarify the inclusion and exclusion criteria; (iv). Clearly define the outcome of the measurement; (v). The data collected should reach the expected goal; (vi). Accurately describe that patients are continuously recruited; (vii). Clearly and accurately describe the main findings of the study; (viii). Hierarchical analyze and report outcomes [1].

\subsection{Data Extraction}

Formulate a standard data extraction table which mainly extract the following:(i) Basic information: first author, follow-up time, sample size, sample source; (ii) patient information: age, gender; (iii) methodological characteristics: type of research design, research quality and etc. (iv) Research results: Hypertension, diabetes, heart disease, cerebrovascular disease, chest pain, acute myocardial injury, heart failure, shock, antiarrhythmic and etc. If documents published in the same unit and the same study at different periods are found, whether they are included was judged at the same time according to the respective sample size and influencing factors of the study (Table 1).

\subsection{Statistical Analysis}

Statistical analysis was performed by employing the Stata14.0 analysis software. First, the original rate was subjected to a double arcsine transformation to ensure it was conform to the normal distribution, and then Meta analysis was conducted on the conversion rate. Finally, using the formula $\mathrm{R}=(\sin (\operatorname{tr} / 2)) 2$ to convert the results to get the final rate $(\mathrm{R})$ and its $95 \% \mathrm{CI}$. The $I^{2}$ test us used to analyze the heterogeneity of the included studies, and it was obtained that $\mathrm{P}>0.1$ was not statistically significant among the studies. If there was no statistical heterogeneity among the results of the studies, a fixed effect model was employed for Meta analysis; if there was statistical heterogeneity among the results of the studies, subgroup analysis and sensitivity analysis are employed to explore the source of heterogeneity. After obvious clinical heterogeneity, a random effect model would be used for Meta analysis. According to the funnel char and combining with the Egger's and Begg's tests to determine whether there exists publication bias.

\section{Result}

\subsection{Literature Inclusion}

A total of 405 articles were retrieved, among which 63 papers were removed due to repeated retrieval, 288 papers were eliminated after reading titles and abstracts, 32 papers were excluded after reading the full text. At the end, a total of 22 articles were included in this Meta-analysis, including data from 3863 patients. The subjects were Chinese, 13 subjects were in Wuhan, and the remaining 9 subjects were outside Wuhan. The specific operation flow was shown in Figure 1. The characteristics of the literature are shown in Table 1.

Table 1. Baseline characteristics of included studies.

\begin{tabular}{|c|c|c|c|c|c|c|c|c|c|}
\hline author & area & $\begin{array}{l}\text { follow-up } \\
\text { time }(2020) \\
\end{array}$ & Mean Age & No. Patients & Female (\%) & Diabetes (\%) & Hypertension (\%) & CHD (\%) & $\begin{array}{l}\text { Literature } \\
\text { Quality }\end{array}$ \\
\hline Chen [2] & chongqing & $1.1 \sim 2.27$ & 46 & 139 & 45.3 & 5.8 & 7.9 & 0 & 7 \\
\hline Xiang [3] & jiangxi & $1.21 \sim 1.27$ & 43 & 49 & 32.7 & 4.1 & 12.2 & 0 & 6 \\
\hline Yun [4] & wuhan & $1.1 \sim 2.15$ & 53 & 476 & 43.1 & 10.3 & 23.7 & 8 & 8 \\
\hline Yang [5] & wenzhou & $1.17-2.10$ & 45 & 149 & 45.6 & 6 & 0 & 18.8 & 6 \\
\hline Jia [6] & qingdao & $1.29 \sim 2.23$ & 46 & 44 & 65.9 & 0 & 0 & 0 & 6 \\
\hline Mo [7] & wuhan & $1.1 \sim 2.5$ & 54 & 155 & 44.5 & 9.7 & 23.9 & 9.7 & 7 \\
\hline $\mathrm{Wu}[8]$ & suzhou & $1.22 \sim 2.14$ & 46 & 80 & 51.3 & 6.3 & 31.3 & 31.3 & 8 \\
\hline Chen [9] & wuhan & $1.1 \sim 2.20$ & 55 & 99 & 32.3 & 13.1 & 40.4 & 40.4 & 6 \\
\hline Wang [10] & wuhan & $01.01 \sim 01.28$ & 56 & 138 & 45.7 & 10.1 & 31.2 & 14.5 & 7 \\
\hline Xiong [11] & wuhan & $01.17 \sim 02.20$ & 53 & 89 & 55.1 & 15.7 & 29.2 & 0 & 6 \\
\hline Zhang [12] & beijing & $1.21 \sim 2.11$ & 52 & 74 & 52.7 & 4.1 & 17.6 & 12.2 & 6 \\
\hline
\end{tabular}




\begin{tabular}{|c|c|c|c|c|c|c|c|c|c|}
\hline author & area & $\begin{array}{l}\text { follow-up } \\
\text { time }(2020)\end{array}$ & Mean Age & No. Patients & Female (\%) & Diabetes (\%) & Hypertension (\%) & CHD (\%) & $\begin{array}{l}\text { Literature } \\
\text { Quality }\end{array}$ \\
\hline Cao [13] & wuhan & $1.3 \sim 2.1$ & 54 & 102 & 47.1 & 10.8 & 27.5 & 4.9 & 6 \\
\hline Zhou [14] & wuhan & $12.29 \sim 1.31$ & 56 & 191 & 37.7 & 18.8 & 30.4 & 7.9 & 6 \\
\hline Wang [15] & wuhan & $1.1 \sim 2.6$ & 69 & 339 & 51 & 15.9 & 40.7 & 15.6 & 7 \\
\hline Lei i [16] & wuhan & $1.1 \sim 2,5$ & 55 & 34 & 58.8 & 23.5 & 38.2 & 20.6 & 6 \\
\hline Shi i [17] & wuhan & $1.20 \sim 2.10$ & 64 & 416 & 50.7 & 14.4 & 30.5 & 10.5 & 7 \\
\hline Guo [18] & wuhan & $1.23 \sim 2.23$ & 58 & 187 & 51.3 & 15 & 32.6 & 11.2 & 7 \\
\hline Li [19] & wuhan & $1.26-2.5$ & 60 & 548 & 49.1 & 15.1 & 30.2 & 6.2 & 7 \\
\hline Liu [20] & hainan & $1.15 \sim 2.18$ & $\mathrm{~N}$ & 54 & 46.3 & 7.4 & 18.5 & 3.7 & 6 \\
\hline Qian [21] & zhejiang & $1.20 \sim 2.11$ & 50 & 91 & 59.3 & 8.8 & 16.5 & 3.3 & 8 \\
\hline Wan [22] & chongqing & $1.23 \sim 2.8$ & 47 & 135 & 46.7 & 8.9 & 9.6 & 5.2 & 8 \\
\hline Chen [23] & wuhan & $1.13 \sim 2.12$ & 62 & 274 & 37.6 & 17.2 & 33.9 & 8.4 & 7 \\
\hline
\end{tabular}

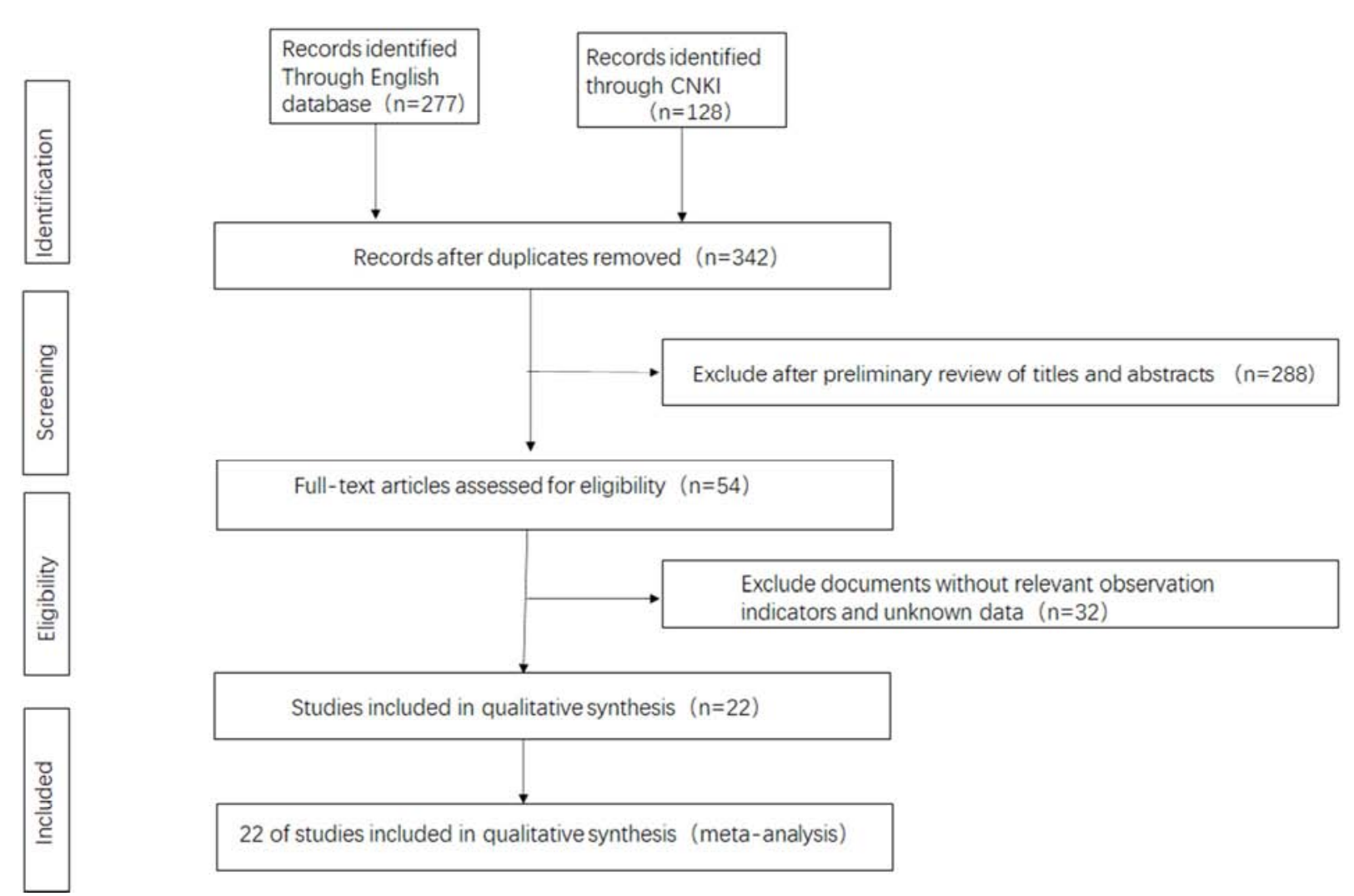

Figure 1. Flow diagram for selection of studies for inclusion in this meta-analysis.

\subsection{Meta Analysis on Acute Myocardial Injury}

Patients with COVID-19 infection have an increase and / or decrease in myocardial injury marker [troponin (cTNI / cTNT or CK-MB)] exceeding the 99th percentile upper limit (URL) without clinical evidence of myocardial ischemia. Moreover, it was likely to be accompanied by elevated levels of B-type natriuretic peptide (BNP) or $\mathrm{N}$-terminal pro-B-type natriuretic peptide (NT-proBNP), which was diagnosed as a new coronavirus infection-related myocardial injury [6], including 9 reports [10-17, 19, 20] (A total of 1985 patients) regarding to the study of acute myocardial injury. Significant statistical heterogeneity was shown among the studies $\left(\mathrm{I}^{2}=87.6 \%\right.$, $P<0.001)$. The random effect model was being made to combine with the effect amount for analysis. The results indicated that the incidence of myocardial injury in patients with new coronavirus infection was $18 \%$, (95\% I: 0.12 , 0.23) (Figure 2A).

\subsection{Meta Analysis on Symptoms of Myocardial Injury}

Ten studies [2-9, 17, 19] (a total of 2155 patients) were included to report the symptoms of chest pain related to myocardial injury associated with novel coronavirus infection. The results show that the incidence of chest pain in infected patients was $4 \%(95 \% \mathrm{CI}$ : 0.02, 0.05) (Figure 2B). Six studies $[2,5,15,19,21,22]$ ( a total of 1401 patients) were included to report the symptoms of chest distress, and the incidence of chest distress in infected patients was $31 \%(95 \% \mathrm{CI}: 0.16,0.47)$ (Figure 2C). Three studies [2, 20, 22] ( a total of 394 
patients) were included to report the symptoms of palpitations, and the incidence of palpitations in infected patients was 4\% (95\%CI: 0.00, 0.07) (Figure 2D).

\subsection{Meta Analysis on Complications of Myocardial Injury}

Three studies [14, 15, 23] on COVID-19 infection with heart failure are included in the report The random effect model was being used to analyze the combined effect size. The results indicate that the incidence rate of new coronavirus infection patients with heart failure was $22 \%$ (95\%CI: 0.17, 0.28) (Figure 3A). Including six studies $[10-13,15,23]$ on the new coronavirus infection combined with shock, using random effects model combined effect analysis, the results convey that the incidence of infected patients with shock was $9 \%(95 \% \mathrm{CI}: 0.03,0.14)$ (Figure 3B). Five studies $[10,12,13,15,16,18]$ on new coronavirus infection combined with arrhythmia are being included in the study. A random effect model was then being used to analyze the combined effects and the results show that the new corona incidence of viral infection patients with arrhythmia was at $13 \%(95 \% \mathrm{CI}: 0.08,0.19)$

\section{(Figure 3C).}

\subsection{Analysis on Sensitivity and Subgroup}

Heterogeneity analysis was performed on acute myocardial injury with high heterogeneity, and the statistics were being combined after excluding each study in turn. The results indicate that there are no directional changes, suggesting that the results were more stable (Figure 4B). A subgroup analysis of the study age of myocardial injury reveals significant heterogeneity in 5 studies over 55 years of age, and no significant statistical heterogeneity in 5 studies under 55 years of age (Figure 4A), showing the age of patients was a relatively important factor in this result for heterogeneity.

\subsection{Publication Bias}

The funnel chart of myocardial injury show that the left and right distribution symmetry of each study site do not reach the expectation (Figure 4C), but the $\mathrm{P}$ value of the Begg's was 0.371 (Figure 4D), suggesting that there was little possibility of publication bias happening in the studies.
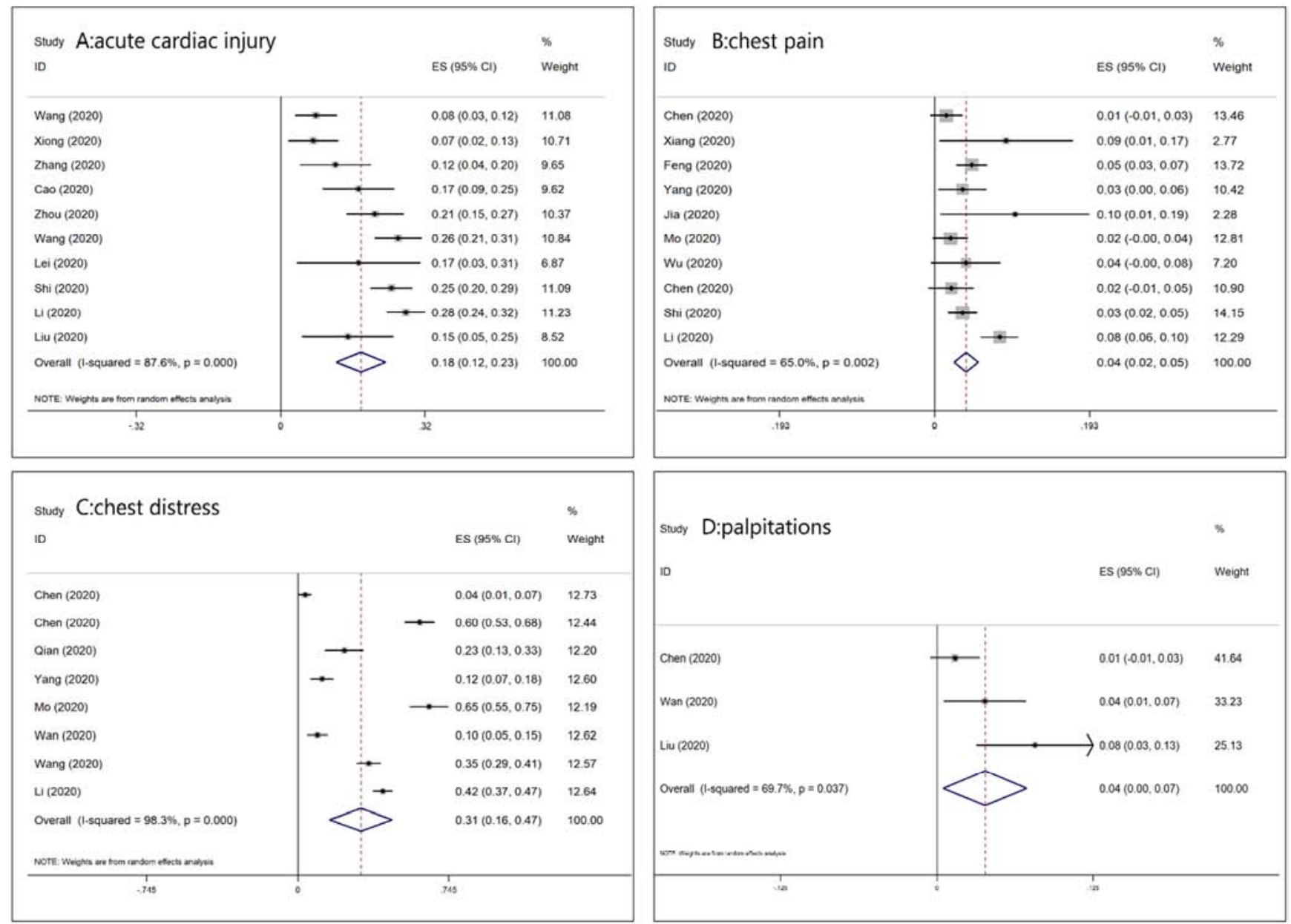

Figure 2. Forest plot of the prevalence of cardiac injury and cardiac symptoms in patients with COVID-19. Weights were calculated from binary random-effects model analysis. (A. acute cardiac injury. B. chest pain. C. chest distress. D. palpitations). CI=confidence interval, COVID-19=Corona Virus Disease 2019. 


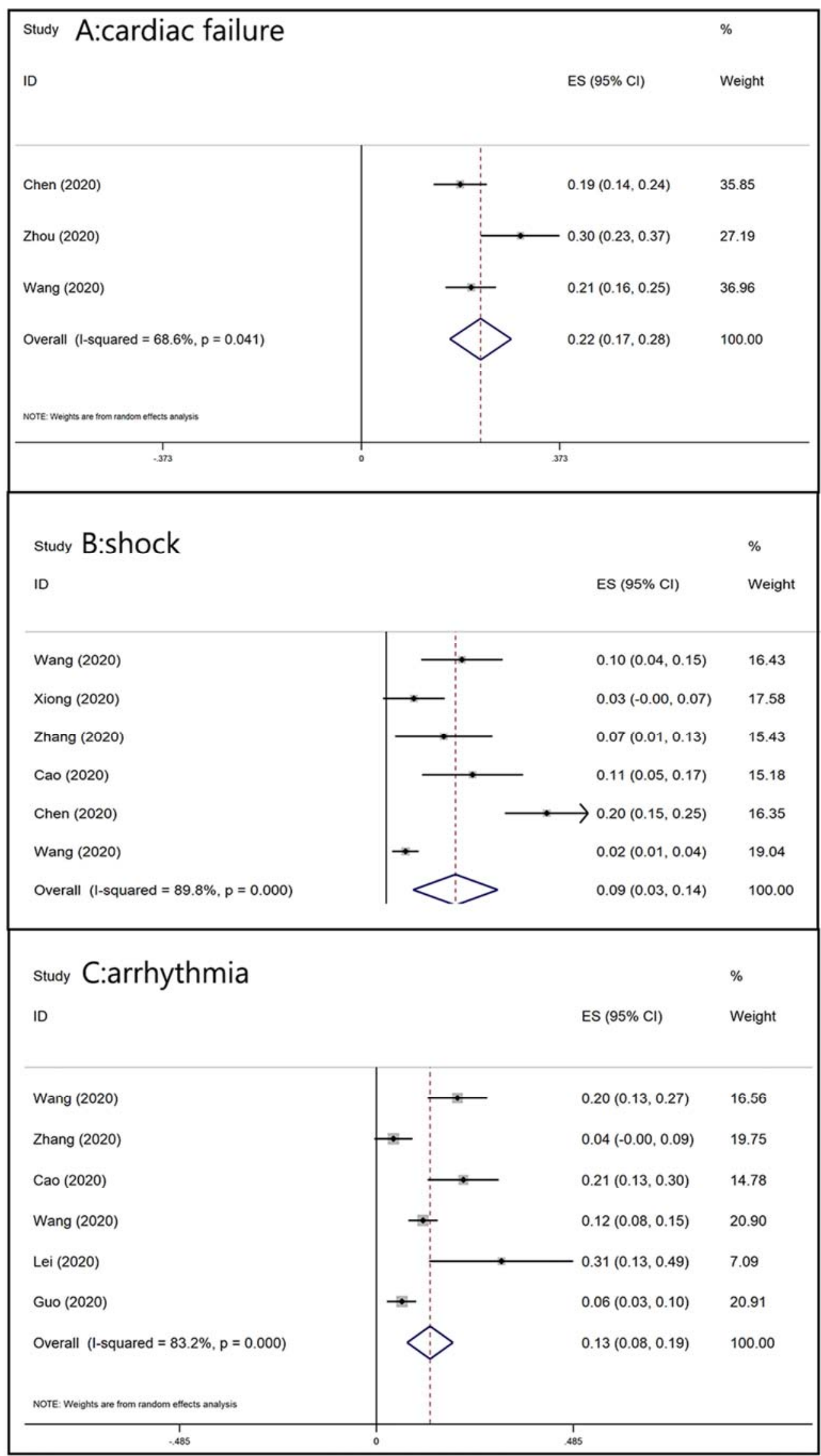

Figure 3. Forest plot of the incidence of complications in patients with COVID-19. (A. cardiac failure. B. shock. C. arrhythmia). 

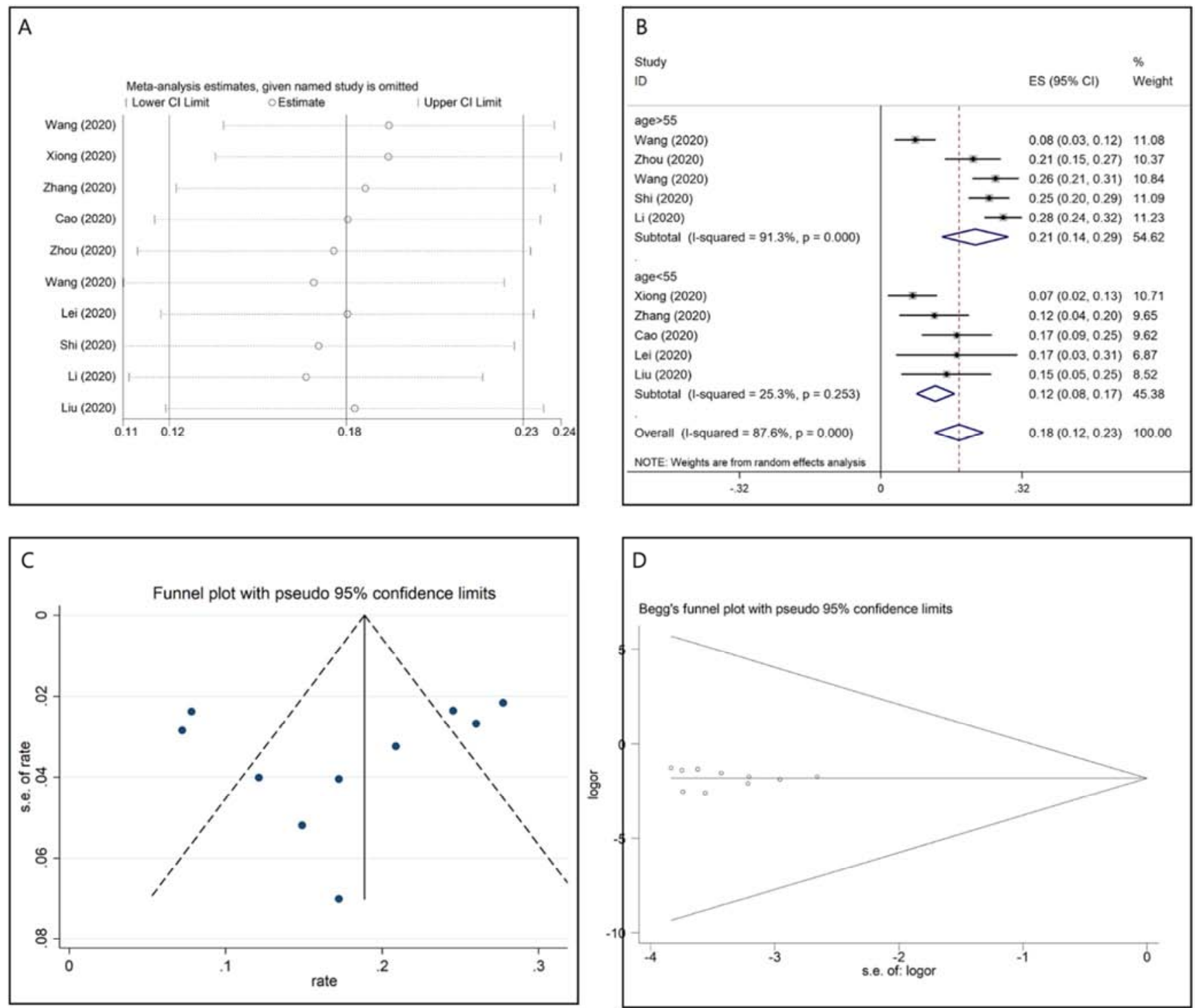

Figure 4. A. Forest plot of subgroup analysis of acute cardiac injury. B. Sensitivity analysis of acute cardiac injury C. Funnel-plot analysis showing asymmetrical funnel plot for acute cardiac injury. D Begg's funnel plot of acute cardiac injury.

\section{Discussion}

Novel coronavirus still of international concern, Various epidemiological studies have shown that severe patients infected with 2019 novel coronavirus have underlying Diseases, such as hypertension diabetes and coronary heart Disease, those patients are in a more dangerous state and have a higher risk of death, suggesting SARS-CoV-2 infection does not only cause myocardial damage, but also may lead to exacerbations of the cardiovascular Disease [24]. COVID-19 and vascular events, vascular dseases are strongly intertwined. An increased risk of vascular events has been observed in COVID-19 patients.

Also, a higher rate of infection with COVID-19, severe COVID-19 and bad outcome has been found in patients with pre-existing vascular dseases. Possible mechanism of myocardial damage caused by SARS-CoV-2: comprising direct viral myocardial damage, down-regulation of ACE2, immune injury and inflammatory storm, imbalance of oxygen supply and demand, drug-induced injury, acute stress, endogenous catecholamine adrenergic status and so on [25]. Heart palpitations, chest tightness, chest pain, difficulty breathing in severe cases, and even lead to heart failure, cardiac arrest, cardiogenic shock [26, 27].

The research includes a total of number of 3863 patients in 22 studies. The meta-analysis results show that the incidence of myocardial injury in patients with an average age of 55 years or less in new coronavirus infection was $12 \%$ which was different from the meta-analysis of people such as Li B [24], showing at least $10.0 \%$ of COVID-19 patients suffer acute heart injury. It was considered that this analysis includes only two studies in the early stage of the outbreak, suggesting that as patients' age and the prolonged duration of the outbreak significantly increases, the probability of myocardial injury would also increase. This study also reveals that the incidence of chest pain in patients with new coronavirus infection was $4 \%$, however, the incidence of chest distress was $31 \%$, There shows significant statistical 
heterogeneity among the studies $\left(\mathrm{I}^{2}=98.3 \%, P<0.001\right)$. We considered that the symptoms of chest distress are not specific to acute myocardial injury associated with COVID-19 infected, some patients with a history of chronic respiratory dseases and those wear surgical masks also cause chest distress. myocardial damage without symptoms of myocardial injury should be highly vigilant in the course of infection.

On the other hand, early diagnosis can be puzzled in the COVID-19 infection patients with chronic cardiac condition, once the most frequent symptoms, like fatigue, dyspnea, and cough can also be manifestations of heart failure. Subsequently, the National Health Commission of China (NHC) reported that cardiovascular symptoms were the first presentation among the some COVID-19 infection confirmed cases. It was noteworthy that rapid diagnosis and appropriate monitoring seem however to improve prognosis. Unfortunately, due to laboratory testing and standardization was not uniform, the lack of detailed data of abnormal cardiac biomarkers, such as Troponin I or T, CK-MB, meant that this part of meta-analysis could not be undertaken.

Arrhythmia could be the first presentation of COVID-19, and progressive and/or new-onset arrhythmia could indicate cardiac injury. This study reveals that palpitations in patients with new coronavirus infection was $4 \%$, however, the incidence of arrhythmia in patients with new coronavirus infection was $13 \%$, But unfortunately, all studies did not specifically describe the type of arrhythmia, and cases of tachycardia caused by high fever cannot be ruled out. Therefore probability of occurrence in the course of that arrhythmia was caused by new coronavirus infection was overestimated. Besides, hypoxemia caused by COVID-19 can trigger atrial fibrillation, which was the most common arrhythmia among elderly patients frequently complicated with a series of basic dseases. Influenza and the exacerbation of ischemia by increased oxygen demand can also play a role in the increase of arrhythmic events among patients with underlying ischemic cardiomyopathy [28]. Last but no least, medical treatement, such as chloroquine, azithromycin, were known to cause cardiovascular side effects including prolongation of QT interval.

SARS-CoV2 infection was supposed to be the main driver of heart failure in cardiovascular dseases with COVID-19. Most current studies have shown that patients with cardiovascular underlying Diseases are associated with significantly increased morbidity and mortality [24]. However, there have also been reports of severe cardiac dysfunction caused by viral myocarditis during the course of previous healthy crown patients [27]. This meta-analysis of complications with COVID-19 shows that the probability of cardiac failure was $22 \%$. Three articles included in this meta-analysis show that the study object was with severe manifestations or a part of them have died from COVID-19 infection. This trend underlines the need to considerate the influence of SARS-CoV2 infection on the cardiovascular system and hemodynamic considerations in patients. The meta-analysis indicates that the incidence of combined shock was $9 \%$, excluding paper such as Chen [23], the heterogeneity decreased, considering that the study object were severe COVID-19 patients with a high mortality rate. Furthermore, these studies did not distinguish cardiogenic shock from septic shock.

This study was also associated with some limitations: (i) There may be a publication bias; only four out of the 22 studies included are multi-center studies whereas the others are single-center studies, and the source of the study was mostly from Wuhan so there may exist admission bias movement and select offset. (ii) Most of the included studies are small sample studies so that the testing efficiency may be insufficient. (iii) The patient's course of Disease and the severity of the Disease are inconsistent, which may lead to clinical heterogeneity. (iv) The included studies are all retrospective studies and therefore the influence of confounding factors could not be controlled.

\section{Conclusion}

COVID-19 interacts with the cardiovascular system at various levels and has caused direct and indirect damage. Acute cardiac injury in patients hospitalized with COVID-19 is associated with higher morbidity and mortality. When a cardiac patient is presenting with fever or other symptoms of infection, a comprehensive evaluation has to be performed. However, acute cardiac injury without clinical symptoms should be paid more attention. In addition. It highlights the need to effectively monitor heart health to prevent the cardiac complications (cardiac failure, shock, arrhythmia) in patients infected with COVID-19.

\section{Funding}

This work was part of the program on science and technology planning project (special medical and health projects) funded under the Science, Technology and Information Bureau of Tianhe District Guangzhou, China, grant number: 2018YT013.

\section{Conflict of Interest}

All the authors do not have any possible conflicts of interest.

\section{References}

[1] Yu H, Yang H, Liu JP (2008) Reporting methods of expert clinical examination and experience-design and quality evaluation of case series. Chinese Medicine Journal (05): 407-410.

[2] Chen X, Tong J, Xiang Jh, Hu JJ. Retrospective study on the epidemiological characteristics of 139 patients with novel coronavirus pneumonia on the effects of Severity. Chongqing Medicine1-9. http://kns.cnki.net/kcms/detail/50.1097. R.20200313.1537.004.html. 
[3] Xiang TX, Liu JM, Xu F, Chen N, Liu Y, Qian KJ, Zhang W. Analysis of clinical characteristics of 49 patients with Novel Coronavirus Pneumonia in Jiangxi province. Chinese Journal of Respiratory and Critical Care Medicine. 2020, 19 (02): 154-160.

[4] Feng Y, Ling Y, Bai T, Xie Y, Huang J, Li J, Xiong W, Yang D, Chen R, Lu F, Lu Y, Liu X, Chen Y, Li X, Li Y, Summah HD, Lin H, Yan J, Zhou M, Lu H, Qu J. COVID-19 with Different Severities: A Multicenter Study of Clinical Features. Am J Respir Crit Care Med. 2020 Jun 1; 201 (11): 1380-1388. doi: 10.1164/rccm.202002-0445OC.

[5] Yang W, Cao Q, Qin L, Wang X, Cheng Z, Pan A, Dai J, Sun Q, Zhao F, Qu J, Yan, F. Clinical characteristics and imaging manifestations of the 2019 novel coronavirus Disease (COVID-19): A multi-center study in Wenzhou city, Zhejiang, China. J Infect, 80 (4): 388-393.

[6] Jia J, Hu X, Yang F, Song X, Dong L, Zhang J, Jiang F, Gao R. Epidemiological Characteristics on the Clustering Nature of COVID-19 in Qingdao City, 2020: A Descriptive Analysis. Disaster Med Public Health Prep. 2020 Mar 31: 1-5. doi: 10.1017/dmp.2020.59.

[7] Mo P, Xing Y, Xiao Y, Deng L, Zhao Q, Wang H, Xiong Y, Cheng Z, Gao S, Liang K, Luo M, Chen T, Song S, Ma Z, Chen X, Zheng R, Cao Q, Wang F, Zhang, Y. Clinical characteristics of refractory COVID-19 pneumonia in Wuhan, China [published online ahead of print, 2020 Mar 16]. Clin Infect Dis. Ciaa 270. doi: 10.1093/cid/ciaa270.

[8] Wu J, Liu J, Zhao X, Liu C, Wang W, Wang D, Xu W, Zhang C, Yu J, Jiang B, Cao H, Li L. Clinical Characteristics of Imported Cases of COVID-19 in Jiangsu Province: A Multicenter Descriptive Study [published online ahead of print, $2020 \mathrm{Feb}$ 29]. Clin Infect Dis. ciaa199. doi: 10.1093/cid/ciaa199.

[9] Chen N, Zhou M, Dong X, Qu J, Gong F, Han Y, Qiu Y, Wang J, Liu Y Wei Y, Xia J, Yu T, Zhang X, Zhang L. Epidemiological and clinical characteristics of 99 cases of 2019 novel coronavirus pneumonia in Wuhan, China: a descriptive study. Lancet. 395 (10223): 507-513. doi: 10.1016/S0140-6736(20)30211-7.

[10] Wang D, Hu B, Hu C, Zhu F, Liu X, Zhang J, Wang B, Xiang $\mathrm{H}$, Cheng Z, Xiong Y, Zhao Y, Li Y, Wang X, Peng Z. Clinical Characteristics of 138 Hospitalized Patients With 2019 Novel Coronavirus-Infected Pneumonia in Wuhan, China. Jama, 323 (11).

[11] Xiong J, Jiang WL, Zhuo Q, Hu XQ, Liu CY. Clinical characteristics, treatment, and prognosis in 89 cases of COVID-2019. Medical Journal of Wuhan University. 2020, 41 (04), 542-546 DOI: 10.14188/j.1671-8852.2020.0103.

[12] Zhang W, Hou W, Li TZ, Li AX, Pan W, Jin RH, Liang LC, $\mathrm{Hu} \mathrm{ZJ}$. Clinical characteristics of 74 hospitalized patients with COVID-19, Journal of Capital Medical University. 2020, $41 \quad$ (2): $161-167 . \quad$ DOI: 10.3969/j.issn.1006-7795.2020.02.003.

[13] Cao J, Tu WJ, Cheng W, Yu L, Liu YK, Hu X, Liu Q. Clinical Features and Short-term Outcomes of 102 Patients with Coronavirus Disease 2019 in Wuhan, China. Clin Infect Dis. 2020 Jul 28; 71 (15): 748-755. doi: $10.1093 / \mathrm{cid} / \mathrm{ciaa} 243$.

[14] Zhou F, Yu T, Du R, Fan G, Liu Y, Liu Z, Xiang J, Wang Y, Song B, Gu X, Guan L, Wei Y, Li H, Wu X, Xu J, Tu S, Zhang $\mathrm{Y}$, Chen $\mathrm{H}$, Cao, B. Clinical course and risk factors for mortality of adult inpatients with COVID-19 in Wuhan, China: a retrospective cohort study. The Lancet, 395 (10229): 1054-1062.

[15] Wang L, He W, Yu X, Hu D, Bao M, Liu H, Zhou J, Jiang H. Coronavirus Disease 2019 in elderly patients: Characteristics and prognostic factors based on 4-week follow-up. J Infect. 2020 Jun; 80 (6): 639-645. doi: 10.1016/j.jinf. 2020.03.019. Epub 2020 Mar 30.

[16] Lei S, Jiang F, Su W, Chen C, Chen J, Mei W, Zhan LY, Jia Y, Zhang L, Liu D, Xia ZY, Xia Z. Clinical characteristics and outcomes of patients undergoing surgeries during the incubation period of COVID-19 infection. EClinicalMedicine. 2020 Apr 5; 21: 100331. doi: 10.1016/j.eclinm.2020.100331. PMID: 32292899; PMCID: PMC7128617.

[17] Shi S, Qin M, Shen B, Cai Y, Liu T, Yang F, Gong W, Liu X, Liang J, Zhao Q, Huang H, Yang B, Huang C. Association of Cardiac Injury With Mortality in Hospitalized Patients With COVID-19 in Wuhan, China. JAMA Cardiol. e200950. doi: 10.1001/jamacardio.2020.0950.

[18] Guo T, Fan Y, Chen M, Wu X, Zhang L, He T, Wang H, Wan J, Wang X, Lu Z. Cardiovascular Implications of Fatal Outcomes of Patients With Coronavirus Disease 2019 (COVID-19). JAMA Cardiol. 2020 Jul 1; 5 (7): 811-818. doi: 10.1001/jamacardio.2020.1017.

[19] Li X, Xu S, Yu M, Wang K, Tao Y, Zhou Y, Shi J, Zhou M, Wu, B, Yang Z, Zhang C, Yue J, Zhang Z, Renz H, Liu X, Xie J, Xie M, Zhao J. Risk factors for severity and mortality in adult COVID-19 inpatients in Wuhan [published online ahead of print, 2020 Apr 12]. J Allergy Clin Immunol. S0091-6749(20)30495-4. doi: 10.1016/j.jaci.2020.04.006.

[20] Liu K, Chen Y, Lin R, Han K. Clinical features of COVID-19 in elderly patients: A comparison with young and middle-aged patients. J Infect. 2020 Jun; 80 (6): e14-e18. doi: 10.1016/j.jinf.2020.03.005. Epub 2020 Mar 27.

[21] Qian GQ, Yang NB, Ding F, Ma AHY, Wang ZY, Shen YF, Shi CW, Lian X. Chu JG, Chen L, Wang ZY, Ren DW, Li GX, Chen XQ, Shen HJ, Chen XM (2020) Epidemiologic and Clinical Characteristics of 91 Hospitalized Patients with COVID-19 in Zhejiang, China: A retrospective, multi-centre case series. QJM. Hcaa 089. doi: 10.1093/qjmed/hcaa089.

[22] Wan S, Xiang Y, Fang W, Zheng Y, Li B, Hu Y, Lang C, Huang D, Sun Q, Xiong Y, Huang X, Lv J, Luo Y, Shen L, Yang H, Huang G, Yang R. Clinical features and treatment of COVID-19 patients in northeast Chongqing. J Med Virol. 2020 Jul; 92 (7): 797-806. doi: 10.1002/jmv.25783.

[23] Chen T, Wu D, Chen H, Yan W, Yang D, Chen G, Ma K, Xu D, Yu H, Wang H, Wang T, Guo W, Chen J, Ding C, Zhang X, Huang J, Han M, Li S, Luo X, Zhao J, Ning Q. Clinical characteristics of 113 deceased patients with coronavirus Disease 2019: retrospective study. Bmj. 368: m1091. Published 2020 Mar 26. doi: 10.1136/bmj.m1091.

[24] Li B, Yang J, Zhao F, Zhi L, Wang X, Liu L, Bi Z, Zhao Y. Prevalence and impact of cardiovascular metabolic Diseases on COVID-19 in China. Clin Res Cardiol. 109 (5): 531-538. doi: 10.1007/s00392-020-01626-9.

[25] Peng W, Gan X, Tan Y, Cao F, Li S. Damage mechanisms of 2019-nCoV infectious Disease to cardiovascular system and advance in treatment. Chin J Nosocomiol. (08): 1177-1182. 
[26] Tavazzi G, Pellegrini C, Maurelli M, Belliato M, Sciutti F, Bottazzi A, Sepe PA, Resasco T, Camporotondo R, Bruno R, Baldanti F, Paolucci S, Pelenghi S, Iotti GA, Mojoli F, Arbustini E. Myocardial localization of coronavirus in COVID-19 cardiogenic shock. Eur J Heart Fail. 2020 May; 22 (5): 911-915. doi: 10.1002/ejhf.1828. Epub 2020 Apr 11. PMID: 32275347; PMCID: PMC7262276.

[27] Inciardi RM, Lupi L, Zaccone G, Italia L, Raffo M, Tomasoni D, Cani DS, Cerini M, Farina D, Gavazzi E, Maroldi R, Adamo M, Ammirati E, Sinagra G, Lombardi CM, Metra M. Cardiac
Involvement in a Patient With Coronavirus Disease 2019 (COVID-19). JAMA Cardiol. 2020 Jul 1; 5 (7): 819-824. doi: 10.1001/jamacardio.2020.1096.

[28] Madjid M, Connolly AT, Nabutovsky Y, Safavi-Naeini P, Razavi M, Miller CC. Effect of high influenza activity on risk of ventricular arrhythmias requiring therapy in patients with implantable cardiac defibrillators and cardiac resynchronization therapy defibrillators. Am J Cardiol. 2019; 124: 44-50. 